\title{
INTERLABORATORY VARIABILITY OF RADIOCARBON RESULTS OBTAINED FROM BLIND AMS ANALYSES ON SEVERAL MODERN CARBON SAMPLES
}

\author{
Glenn A Norton \\ Center for Industrial Research and Service, Iowa State University, Ames, Iowa 50011, USA. Email: nortong@iastate.edu.
}

\begin{abstract}
Three samples of modern-day vegetation collected in 2009-2010 and a sample of bioethanol produced in 2010 were analyzed for radiocarbon by 5 different accelerator mass spectrometry (AMS) laboratories in a blind analysis study. The magnitude of any variability in the reported results for percent modern carbon (pMC) was observed. Results indicated that the interlaboratory repeatability on the samples of vegetation was generally very good, varying by no more than $\sim 1 \mathrm{pMC}$ for 2 of the 3 samples. Results for the bioethanol were less consistent, and varied by $5.5 \mathrm{pMC}$ (ranging from 101.9 to $107.4 \mathrm{pMC}$ ). Variations in the $\delta^{13} \mathrm{C}$ values used to correct for isotopic fractionation did not account for the variability observed in the pMC values for this sample. In view of the homogeneity of the bioethanol and its inherent simplicity in composition, this suggests that volatile liquid fuels may be more difficult to prepare for analysis without incurring significant sample processing errors. When viewing all of the results as a whole, the analytical errors (incorporating both instrumental and sample processing errors) appeared to be more random than systematic in nature. Because of analytical uncertainties in pMC measurements, as well as inherent local and regional variations in ${ }^{14} \mathrm{C}$ activity levels known to occur in modern-day biomass, there is not a precise (accurate to 2 decimal places) correction factor for negating the bomb carbon effect that is applicable to all biofuels or other biobased products being analyzed in accordance with ASTM Method D6866. Therefore, a reasonable correction factor (currently set at 0.95 ) needs to be consistently applied in order to make comparisons of biobased content data from different laboratories more valid. Results from this study indicate that, for samples containing predominantly modern carbon, reporting results to the nearest $0.1 \mathrm{pMC}$ is not warranted.
\end{abstract}

\section{INTRODUCTION}

Radiocarbon $\left({ }^{14} \mathrm{C}\right)$ analyses are commonly employed to determine the "biobased content" of liquid fuels and other commercial products using ASTM Method D6866 (ASTM International 2010). The biobased content is defined as the percentage (mass) of the organic carbon that is derived from modern-day plants or animals. Unlike ${ }^{14} \mathrm{C}$ analyses for dating applications, where "modern" carbon has a very precise definition, the term "modern" does not have a precise definition for biobased content testing performed in accordance with Method D6866. The method assumes that all of the organic carbon in a product is either fossil in origin or modern in origin, where "modern" is generally taken to refer to carbon from organisms less than about $5 \mathrm{yr}$ old. That assumption holds true in the vast majority of commercial biobased products.

When performing biobased content determinations, the ${ }^{14} \mathrm{C}$ "bomb pulse" resulting from atomic bomb testing in the late 1950s to mid-1960s must be considered. It is widely known that the bomb testing resulted in substantially higher levels of ${ }^{14} \mathrm{C}$ in the atmosphere, increasing by as much as 70 100\% above 1950 levels between the years 1963 and 1965 (Hua and Barbetti 2004). Since that time, the atmospheric ${ }^{14} \mathrm{C}$ activity level has been steadily decreasing, but has not yet reached an equilibrium level. As a result of this "bomb pulse," modern-day biomass has ${ }^{14} \mathrm{C}$ levels that are significantly higher than the 1950 activity level of $13.56 \mathrm{dpm} /$ gram carbon that represents $100 \mathrm{pMC}$ (percent modern carbon) using conventional ${ }^{14} \mathrm{C}$ reporting procedures. Because of the bomb carbon contribution, a product whose organic carbon is derived entirely from modern biomass will have a reported biobased content of $105 \%$ or more when referenced against $1950{ }^{14} \mathrm{C}$ activity levels. This requires that a correction factor be applied to negate the bomb carbon contribution, such that a biobased product with only modern-day carbon will be reported as having a biobased content close to $100 \%$.

For the applications intended for Method D6866, it is important to specify the bomb correction factor that must be used when analyzing products for biobased content. This provides greater consistency and makes data comparisons between laboratories more valid. After requesting input from 
several ${ }^{14} \mathrm{C}$ laboratories, and in view of data on ${ }^{14} \mathrm{C}$ activity levels in atmospheric $\mathrm{CO}_{2}$ and modernday biomass that were being prepared for presentation at the time (later published by Noakes et al. 2006), the ASTM subcommittee established a correction factor of 0.93 (corresponding to a pMC level of 107.5) in 2004. As such, the pMC value for a given product was multiplied by 0.93 to obtain the product's true biobased content. However, that correction factor requires periodic review since the concentration of bomb carbon in the atmosphere is slowly diminishing with time. It was observed that numerous biobased products containing only "botanical" components produced in the 2009-2010 timeframe were being reported as having a biobased content of 97-99\%. Although this suggested that the bomb carbon correction factor may need to be adjusted, definitive conclusions could not be drawn based solely on those observations. Additional support for adjusting the correction factor came from the fact that several ${ }^{14} \mathrm{C}$ experts believed that $\mathrm{pMC}$ values for present-day (as of 2009-2010) carbon were now less than $107 \mathrm{pMC}$. Opinions varied slightly regarding the precise $\mathrm{pMC}$ value associated with present-day carbon, but the opinions all indicated that a correction factor in the range of 0.94 to 0.96 would be appropriate. Based on those opinions, the ASTM subcommittee established an intermediate value of 0.95 as a revised correction factor to be used for biobased content measurements performed in accordance with the 2010 version of Method D6866.

The slightly different opinions on current pMC levels could be due to analytical errors (and possibly a systematic bias) specific to any given laboratory, or to true differences in $\mathrm{pMC}$ values associated with the specific samples being analyzed by each of the respective laboratories. If different laboratories report significantly different $\mathrm{pMC}$ values on identical samples, this would suggest that one or more of the laboratories may have significant analytical errors in their pMC measurements. Alternatively, if the laboratories each report a nearly identical pMC result for any given sample, but report different $\mathrm{pMC}$ results between samples, this would suggest that regional or site-specific factors associated with the samples come into play. In order to shed some light on these issues, a small "blind analysis" study was performed to examine interlaboratory variability in pMC measurements on present-day biobased materials. A comparison of facilities, equipment, or specific procedures used by different laboratories is not within the scope of this paper, nor is the intent of the paper to help define current $\mathrm{pMC}$ levels or the most appropriate bomb carbon correction factor to use when performing analyses in accordance with Method D6866.

\section{EXPERIMENTAL}

\section{Samples}

In order to acquire information about the analytical variability associated with measuring ${ }^{14} \mathrm{C}$ activity levels, 4 samples of present-day biobased materials were collected. The 4 samples consisted of seeds from prickly ash berries (Xanthoxylum americanum), leaves from wild black raspberry plants (Rubus occidentalis), leaves from "burning bush" shrubs (Euonymus alatus), and bioethanol derived from recent corn crops. Although the primary purpose of these sample submissions was to examine the variability in pMC results obtained from different analytical laboratories, the bioethanol was analyzed 3 times (submitted as separate blind samples) by 2 of the testing laboratories in order to obtain some information on the analytical repeatability within a given laboratory. Comparing the analytical repeatability for all of the laboratories performing the analyses was beyond the scope of this investigation.

The Xanthoxylum americanum berries and Rubus occidentalis leaves were collected from the Lost Lake area of Ledges State Park in February 2009 and July 2009, respectively. Ledges State Park is in a sparsely populated rural area near Boone, Iowa (USA), and Lost Lake itself is in a pristine area 
that is only accessible by foot. Lost Lake is 0.35 miles from the nearest access road and 1 mile from the nearest county highway, both of which are lightly traveled. Therefore, vegetation from the Lost Lake area has little or no carbon "contamination" from automotive exhaust. In addition, no insecticides are used in the park, and herbicides are used only very sporadically for highly localized spot treatments (stumps of freshly cut Autumn Olive shrubs). Furthermore, there is no industry and no power plants in the region. The nearest town is Boone, which is a nonindustrial town 4 miles away with a population of about 13,000 .

The Euonymus alatus ("burning bush") leaves were collected at the edge of a local mall parking lot in October 2010. Because the mall is located in a town of just roughly 55,000 people, the exposure of the vegetation to fossil carbon from automotive exhausts in the vicinity of the mall was not believed to be very substantial. Nonetheless, because some automotive exhaust was still present year-round, this sample was collected to see if there was any indication of lower ${ }^{14} \mathrm{C}$ activity levels in the vicinity of the mall relative to the 2 samples collected in a pristine area.

The bioethanol sample was obtained from a dry-mill corn ethanol plant near Nevada, Iowa, in January 2010. The corn used to produce the ethanol was no more than a year old. In-house analyses using gas chromatography at the ethanol plant indicated that the bioethanol was $98.5 \%$ pure, with water being the primary impurity. On a moisture-free basis, the bioethanol was $99.8 \%$ pure, with the remaining $0.2 \%$ consisting mostly of biobased aromatic compounds produced during the fermentation process. No processing of this sample was necessary prior to submission to the analytical laboratories.

The berries collected near Lost Lake were washed with water, mashed in a porcelain mortar and pestle, and then dried overnight at $40{ }^{\circ} \mathrm{C}$. The dried material was gently rubbed across a clean 5-mesh stainless steel sieve to remove the bulk of the fleshy material. The seeds, which were roughly $3 \mathrm{~mm}$ in length, were then isolated using 8-mesh and 20-mesh stainless steel sieves that had been thoroughly washed and dried prior to use. The seeds were further isolated by using tweezers to pick out the small amounts of residual fleshy material from the seeds.

The Rubus occidentalis and Euonymus alatus leaves appeared to be clean at the time of collection, but were thoroughly rinsed with distilled water as a precaution. After rinsing the leaves, they were air-dried for 2 days and then dried at $40^{\circ} \mathrm{C}$ overnight. The dried leaves were subsequently processed in a Cole-Parmer Model 4301 Analytical Mill to reduce the particle size to -30 mesh. The analytical mill and sieve used to process the samples were thoroughly washed with water and then air-dried prior to processing each sample.

The samples were submitted as blind samples to 5 different accelerator mass spectrometry (AMS) laboratories (each having their own on-site AMS facility) for ${ }^{14} \mathrm{C}$ analyses, and all of the laboratories were instructed to combust the samples without any pretreatment procedures. Since the samples of vegetation were collected above ground and thoroughly washed (even though appearing clean at the time of collection), chemical pretreatments prior to sample combustion were not believed to be warranted. The sample size to be combusted was left to the discretion of each laboratory, since such judgment calls are always required for any sample. All results were corrected for isotopic fractionation and were reported using conventional procedures established by Stuiver and Polach (1977).

\section{RESULTS AND DISCUSSION}

The pMC results obtained by AMS are shown in Table 1, while the $\delta^{13} \mathrm{C}$ values used to correct for isotopic fractionation are shown in Table 2 . All the pMC values had a reported statistical uncertainty 
of \pm 0.4 to $0.6 \mathrm{pMC}$, with the exception of the results from Laboratory 2, which had a reported statistical uncertainty of $\pm 1 \mathrm{pMC}$. However, it is not clear whether the approach used to calculate the statistical uncertainty was the same for each laboratory. The data in Table 1 show that the reported pMC values (corrected for isotopic fractionation) for the Euonymus alatus leaves and the Xanthoxylum americanum seeds were in excellent agreement between laboratories, varying by a maximum of only about $1 \mathrm{pMC}$. The pMC values for the Rubus occidentalis leaves and the bioethanol samples exhibited greater variability, varying by 2.7 and $5.5 \mathrm{pMC}$, respectively. It is of interest that the ethanol sample exhibited the greatest variability between laboratories (ranging from 101.9 to 107.4 pMC), since compositionally this is the least complex of the submitted samples.

Table 1 Summary of pMC values obtained from AMS analyses on biomass and bioethanol samples. ${ }^{\mathrm{a}}$

\begin{tabular}{lllllll} 
Sample & Lab 1 & Lab 2 & Lab 3 & Lab 4 & Lab 5 & Range \\
\hline Xanthoxylum americanum seeds & 106.0 & 105.2 & 104.9 & 104.8 & 105.0 & 1.2 \\
Rubus occidentalis leaves & 104.3 & 106.2 & 104.7 & 104.0 & 103.5 & 2.7 \\
Euonymus alatus leaves & 104.0 & 104.2 & 103.1 & 103.4 & 102.9 & 1.3 \\
Bioethanol & 105.2 & 101.9 & 105.8 & 107.4 & 103.7 & 5.5 \\
& 105.1 & 104.5 & & & & \\
\hline
\end{tabular}

${ }^{a}$ All the pMC values had a reported statistical uncertainty of \pm 0.4 to $0.6 \mathrm{pMC}$, with the exception of Laboratory 2 , which had a reported statistical uncertainty of $\pm 1 \mathrm{pMC}$ for all results.

Table 2 Summary of $\delta^{13} \mathrm{C}$ values (\%o) obtained from AMS analyses on biomass and bioethanol samples.

\begin{tabular}{lrrrrrl}
\hline Sample & Lab 1 & Lab 2 & Lab 3 & Lab 4 & Lab 5 & Range \\
\hline Xanthoxylum americanum seeds & -26.5 & -26.7 & -31.4 & -25.9 & -27.1 & 5.5 \\
Rubus occidentalis leaves & -29.8 & -29.9 & -30.5 & -29.7 & -29.8 & 0.7 \\
Euonymus alatus leaves & -28.2 & -28.0 & -29.3 & -27.9 & -27.9 & 1.4 \\
Bioethanol & -13.1 & -10.4 & -7.1 & -13.0 & -12.4 & 6.0 \\
& -11.6 & -12.2 & & & & \\
\hline
\end{tabular}

For a given sample, any interlaboratory variability in the measured $\delta^{13} \mathrm{C}$ values could lead to variability in the reported pMC results. As noted above, the largest variations in pMC values reported for a given sample were for the black raspberry (Rubus occidental) leaves and the bioethanol. For the black raspberry leaves, the $\delta^{13} \mathrm{C}$ values reported by the different laboratories were essentially identical to one another, and therefore had no impact on the variability noted in the pMC results for that sample. On the other hand, the $\delta^{13} \mathrm{C}$ values reported for the bioethanol varied by $6 \%$. That degree of variability in the $\delta^{13} \mathrm{C}$ values could cause a variability of up to $1 \mathrm{pMC}$. However, it is clear that this is not the primary cause of the variability in the reported pMC values for the bioethanol. This suggests that volatile liquid fuels may be more difficult to prepare for analysis without incurring significant sample processing errors. If the variability in $\mathrm{pMC}$ values noted for the bioethanol are truly due to sample processing errors, then using the direct liquid scintillation counting (LSC) approach for analyzing liquid fuels (Dijs et al. 2006; Norton 2009) might reduce the amount of interlaboratory variability for those types of samples, since that method does not involve sample combustions with subsequent processing of $\mathrm{CO}_{2}$. However, the interlaboratory repeatability of the direct LSC method has not yet been explored. 
For any given laboratory, all of the pMC results for the Euonymus alatus leaves collected from a mall parking lot were consistently lower (generally by $2 \mathrm{pMC}$ ) than the results for the Xanthoxylum americanum seeds collected in a pristine area. This would presumably be due to the fact that the leaves collected from a mall parking lot were exposed to automotive emissions much of the time. Relative to the Rubus occidental leaves, the pMC values were also always lower for the vegetation collected in a mall parking lot, but the magnitude of the decrease was less consistent and was often well within the statistical uncertainties reported by the laboratories.

For the samples of vegetation collected in a pristine area, all of the pMC results were in the range of 104 to $106 \mathrm{pMC}$. Although these results were not used to help determine the bomb carbon correction factor of 0.95 recently stipulated in Method D6866, they support the use of that correction factor. Similarly, the average pMC value for analyses on all of the samples was $104.6 \pm 1.2 \mathrm{pMC}$. This average value also supports using a correction factor of 0.95 . Although the average value incorporates a sample that had some exposure to automotive exhausts, it is important to note that not all components of biobased products or feedstock materials used to produce biofuels are going to be grown in pristine areas free from petroleum-based contaminants.

In view of the fact that the pMC results ranged from 101.9 to 107.4 for all of the samples analyzed, some of the difficulty in establishing a precise (to 2 decimal places) bomb correction factor is evident. Analytical errors appear to have a significant impact in this regard. The overall results also suggest that, at least for samples with high biobased contents, it is not very meaningful to report results to the nearest $0.1 \mathrm{pMC}$.

In the direct LSC analysis (no sample combustions) of liquid fuel blends containing both petroleumbased and biobased components, using a pure modern-day biofuel (e.g. bioethanol) as an analytical reference sample could potentially eliminate the need for a bomb carbon correction factor, since the ${ }^{14} \mathrm{C}$ activity levels in blended fuels would all be referenced to that sample (resulting in a "percent biofuel" value rather than a "pMC" value). However, local and regional variations in ${ }^{14} \mathrm{C}$ activity levels can occur due to both natural and anthropogenic causes. A well-known example of an anthropogenic cause is the Suess effect, which involves the dilution of atmospheric ${ }^{14} \mathrm{C}$ with carbon from fossil fuel emissions (Tans at al. 1979). In turn, this can result in slightly lower ${ }^{14} \mathrm{C}$ activity levels in biomass that acquired its carbon from atmospheric $\mathrm{CO}_{2}$. It then follows that there may be some variability in ${ }^{14} \mathrm{C}$ activity levels of different liquid biofuels due to variations in the activity levels of the feedstock materials used to produce those fuels. AMS analyses could potentially be used to help verify that the fuel being used as an analytical reference sample does not contain significant levels of petroleum fuel present as contaminants. However, the results shown in Table 1 indicate that there could be a significant amount of uncertainty associated with the AMS analyses on liquid fuels, and therefore such analyses may not provide conclusive information.

\section{CONCLUSIONS}

Blind analyses on a variety of biobased materials indicated that the interlaboratory reproducibility in pMC results was generally very good. The most notable exception was the pMC results reported for the bioethanol sample. In view of the homogeneity of the sample and its inherent simplicity in composition, this suggests that sample processing errors may be involved. The analytical errors, which incorporate both instrumental and sample processing errors, appear to be more random than systematic in nature, since none of the reported values for a given laboratory were consistently higher or lower than those reported by the other laboratories. Because of analytical uncertainties in pMC measurements, as well as inherent local and regional variations in ${ }^{14} \mathrm{C}$ activity levels known to occur in 
modern-day biomass, there is not a precise (accurate to 2 decimal places) bomb correction factor that is applicable to all biobased products (including biofuels). Further blind studies of interlaboratory reproducibility in pMC values would be of interest, particularly for liquid biofuels. However, even the limited amount of data presented in this paper is useful in providing some information on interlaboratory reproducibility when performing blind testing on modern carbon samples.

\section{ACKNOWLEDGMENTS}

This work was supported by a grant from the ConocoPhillips Company.

\section{REFERENCES}

ASTM International. 2010. Method D 6866-10: Standard test methods for determining the biobased content of solid, liquid, and gaseous samples using radiocarbon analysis. ASTM International, 100 Bar Harbor Drive, West Conshohocken, Pennsylvania, USA.

Dijs IJ, van der Windt E, Kaihola L, Van der Borg K. 2006. Quantitative determination by ${ }^{14} \mathrm{C}$ analysis of the biological component in fuels. Radiocarbon 48(3): 315-23.

Hua Q, Barbetti M. 2004. Review of tropospheric bomb radiocarbon data for carbon cycle modeling and age calibration purposes. Radiocarbon 46(3):1273-98.

Noakes J, Norton G, Culp R, Nigam M, Dvoracek D.
2006. A comparison of analytical methods for the certification of biobased products. In: Chałupnik S, Schönhofer F, Noakes J, editors. LSC 2005, Advances in Liquid Scintillation Spectrometry. Tucson: Radiocarbon. p 259-71.

Norton GA. 2009. Direct analysis of automotive fuels for bioethanol content using radiocarbon analysis. Radiocarbon 51(3):995-1003.

Stuiver M, Polach HA. 1977. Discussion: reporting of ${ }^{14} \mathrm{C}$ data. Radiocarbon 19(3):355-63.

Tans PP, de Jong AFM, Mook WG. 1979. Natural atmospheric ${ }^{14} \mathrm{C}$ variation and the Suess effect. Nature 280(5725):826-8. 